\title{
Fungos micorrízicos associados a orquídeas em campos rupestres na região do Quadrilátero Ferrífero, MG, Brasil
}

\author{
Ricardo Eustáquio Nogueira ${ }^{1}$, Olinto Liparini Pereira ${ }^{1}$, Maria Catarina Megumi Kasuya ${ }^{1,4}$, \\ Maria Célia da Silva Lanna² e Míriam Pimentel Mendonça ${ }^{3}$
}

Recebido em 04/06/2004. Aceito em 29/10/2004

\begin{abstract}
RESUMO - (Fungos Micorrízicos associados a orquídeas em campos rupestres na Região do Quadrilátero Ferrífero, MG, Brasil). Oito isolados de fungos micorrízicos rizoctonióides foram obtidos do sistema radicular de orquídeas neotropicais, a saber: Bulbophyllum weddelii (Lindl.) Rchb. f., Epidendrum dendrobioides Thunb., Maxillaria acicularis Herb. ex Lindl., Oncidium gracile Lindl., Pleurothallis teres Lindl., Prosthechea vespa (Vell.) W.E. Higgins, Sophronitis milleri (Blumensch. ex Pabst) C. Berg \& M.W. Chase e Sarcoglottis sp., que ocorrem em campos rupestres da região do Quadrilátero Ferrífero, no Estado de Minas Gerais, Brasil. Três gêneros anamórficos foram identificados: Epulorhiza, isolados do sistema radicular de E. dendrobioides e S. milleri; Ceratorhiza, isolados de B. weddelii, O. gracile, P. teres e P. vespa e Rhizoctonia, isolados de M. acicularis e Sarcoglottis sp. O trabalho constitui-se no primeiro relato taxonômico e de caracterização morfológica de fungos micorrízicos rizoctonióides associados a espécies de orquídeas que ocorrem em campos rupestres no Brasil.
\end{abstract}

Palavras-chave: diversidade, fungos rizoctonióides, Orchidaceae, simbiose

\begin{abstract}
Mycorrhizal fungi associated to orchids growing in "campos rupestres" in "Quadrilátero Ferrífero" region, Minas Gerais State, Brazil). Eight strains of Rhizoctonia-like mycorrhizal fungal were isolated from the root system of neotropical orchids, Bulbophyllum weddelii (Lindl.) Rchb. f., Epidendrum dendrobioides Thunb., Maxillaria acicularis Herb. ex Lindl., Oncidium gracile Lindl., Pleurothallis teres Lindl., Prosthechea vespa (Vell.) W.E. Higgins, Sophronitis milleri (Blumensch. ex Pabst) C. Berg \& M.W. Chase and Sarcoglottis sp., growing in "campos rupestres" in "Quadrilátero Ferrífero" region, Minas Gerais State, Brazil. Three anamorphic genera were identified: Epulorhiza, isolated from the root system of E. dendrobioides and S. milleri; Ceratorhiza, isolated from B. weddelii, O. gracile, P. teres and P. vespa; Rhizoctonia, isolated from M. acicularis and Sarcoglottis sp. This is the first report of Rhizoctonia-like fungi isolated from the root systems of orchids from the "campos rupestres" in Brazil.
\end{abstract}

Key words: diversity, Rhizoctonia-like fungi, Orchidaceae, symbiosis

\section{Introdução}

Dentre as vegetações brasileiras, os campos rupestres destacam-se devido à natureza peculiar de suas partes componentes. Eles ocorrem a partir de uma altitude de $900 \mathrm{~m}$ acima do nível do mar e estão em grande parte associados à Cadeia do Espinhaço, nos Estados de Minas Gerais, Bahia e em disjunções da mesma cadeia entre os limites latitudinais de $21^{\circ} 10^{\prime}$ e $10^{\circ} \mathrm{S}$ (Giulietti \& Pirani 1988).

As porções montanhosas com o seu clima especial, relevo e condições de solo, oferecem as condições para uma flora típica que não é encontrada em outra região do Brasil (Giulitti \& Pirani 1988). As plantas crescem em rochas ou pedras (epilíticas), em solos jovens ou em solos arenosos. As plantas epilíticas têm adaptações para explorar o orvalho, seja por meio da base de folhas velhas, como ocorre em Velloziaceae (Menezes \& Giulietti 1986); por meio de tricomas especializados, como em algumas Bromeliaceae, ou por raízes especializadas e pseudobulbos, como em Orchidaceae (Giulietti \& Pirani 1988).

O Quadrilátero Ferrífero, área de grande representatividade em campos rupestres, é considerado como de "importância biológica extrema" para a biodiversidade do Estado de Minas Gerais (Costa et al. 1998). Dentre as principais razões de tal classificação, foram apontadas a presença de espécies vegetais

\footnotetext{
1 Universidade Federal de Viçosa, Departamento de Microbiologia, Instituto de Biotecnologia Aplicada à Agropecuária, CEP 36570-000, Viçosa, MG, Brasil (liparini@bol.com.br)

2 Universidade Federal de Ouro Preto, Departamento de Ciências Biológicas, Instituto de Ciências Exatas e Biológicas, CEP 35400-000, Ouro Preto, MG, Brasil

3 Jardim Botânico de Belo Horizonte, Fundação Zoo-Botânica, CEP 31365-450, Belo Horizonte, MG, Brasil

4 Autor para correspondência: mkasuya@ufv.br
} 
ameaçadas e endêmicas. $\mathrm{O}$ desmatamento, a expansão urbana, a mineração e o turismo são apontados como as principais pressões antrópicas no Quadrilátero Ferrífero, sendo atualmente recomendado o inventário biológico, a recuperação de áreas degradadas e o monitoramento de espécies ameaçadas, entre outras medidas, para a reabilitação dos campos rupestres da região (Costa et al. 1998).

Há registros desde 1889 de que, na natureza, as orquídeas estão associadas com fungos micorrízicos (Clements 1988). As espécies deste grupo apresentam uma característica única entre as angiospermas: uma estrutura parenquimatosa, o protocórmio, que está intercalada entre o estádio de semente e plântula, e que utiliza a digestão enzimática de fungos simbiontes como meio alternativo para a obtenção de energia, fenômeno denominado micotrofismo (Clements 1988; Rasmussen 1995; Peterson et al. 1998; Pereira et al. 2003b). O micotrofismo pode, em parte, ser responsável pelo sucesso da família Orchidaceae (Smith 1966).

As espécies possuem sementes diminutas, com pouca reserva nutricional e devem ser colonizadas pelo fungo simbionte, como regra, para que ocorra a germinação na natureza (Rasmussen 1995; Peterson et al. 1998; Pereira et al. 2003b). Após a infecção, ocorre a formação de uma estrutura intracelular denominada pelotão. Os produtos advindos da digestão enzimática desta estrutura são utilizados para o desenvolvimento do protocórmio, a partir do embrião contido na semente madura (Peterson et al. 1998; Pereira et al. 2003b). Esses eventos caracterizam o micoheterotrofismo nesta família, ou seja, o protocórmio é totalmente dependente de um fungo simbionte para o seu desenvolvimento até tornar-se plântula autotrófica (Pereira et al. 2003b).

Os floricultores rotineiramente propagam orquídeas germinando sementes em um meio asséptico, seguindo procedimentos inicialmente desenvolvidos por Lewis Knudson na década de 1920 (Arditti et al. 1990). Como resultado, a simbiose micorrízica em Orchidaceae mantém-se pobremente compreendida, quando comparada a outros aspectos fundamentais da biologia de Orchidaceae, a exemplo da sua taxonomia (Rasmunssen 1995). Registros que descrevem os fungos endofíticos de orquídeas do Novo Mundo são raros, particularmente para táxons epífitos ou epilíticos (Richardson et al. 1993; Richardson \& Currah 1995; Pereira et al. 2003a; 2003b; 2005).

No contexto da necessidade de preservação desses ambientes e de sua flora e considerando-se a ausência de registros ou trabalhos envolvendo as interações micorrízicas em orquídeas em campos rupestres, desenvolveu-se este trabalho que teve como objetivo o isolamento, a identificação e a caracterização morfológica de fungos micorrízicos associados a orquídeas em diferentes microhábitats e afloramentos quartzíticos, na região do Quadrilátero Ferrífero, Minas Gerais, Brasil.

\section{Material e métodos}

Os experimentos foram conduzidos no Laboratório de Microbiologia Geral do Departamento de Ciências Biológicas, Núcleo de Pesquisas em Ciências Biológicas, Universidade Federal de Ouro Preto e no Laboratório de Associações Micorrízicas do Departamento de Microbiologia, Instituto de Biotecnologia Aplicada a Agropecuária, Universidade Federal de Viçosa.

Áreas de amostragem - Escolheram-se cinco áreas localizadas em três municípios da Região do Quadrilátero Ferrífero. Em Ouro Preto, foram amostradas áreas no Bairro Morro São Sebastião, no Campus Universitário e na Estrada Real. Em Nova Lima e Belo Vale foram amostradas áreas às margens da Rodovia BR-040. Utilizou-se, como critério para a definição das áreas, a presença de algum empreendimento que estivesse ou pudesse gerar o declínio de populações locais de orquídeas, além, é claro, da obrigatoriedade de serem áreas de campos rupestres, degradados ou não (Tab. 1). Devido à coleta indiscriminada e predatória dessas espécies de orquídeas, para fins horticulturais, não serão fornecidos dados mais precisos sobre a localização dessas populações.

Espécies de orquídeas estudadas - As espécies de orquídeas utilizadas no estudo, assim como os respectivos locais e a caracterização das áreas de coletas, estão apresentados na Tab. 1 .

Isolamento - Amostras das raízes de orquídeas (Tab. 1) foram lavadas em água de torneira corrente por $10 \mathrm{~min}$, cortadas em fragmentos de $2-3 \mathrm{~cm}$ e desinfestadas superficialmente, por imersão em etanol $70 \%$ durante 1 min, e 5 min em solução de hipoclorito de sódio $2 \%$, seguindo-se de cinco lavagens sucessivas em água destilada esterilizada. $\mathrm{O}$ velame foi retirado com bisturi estéril e as raízes foram maceradas em almofariz de porcelana, previamente autoclavado. $\mathrm{O}$ macerado foi espalhado superficialmente em placa de Petri contendo $10 \mathrm{~mL}$ de meio batata-dextrose-ágar 
(BDA). As placas foram incubadas a $28{ }^{\circ} \mathrm{C}$ e observadas diariamente sob microscópio óptico invertido para o isolamento direto do micélio crescido a partir do pelotão (Pereira et al. 2003a), efetuando-se a transferência para outra placa contendo meio MelinNorkran's modificado (MNM) (Marx 1969). Culturas estoques foram mantidas em BDA e discos de $9 \mathrm{~mm}$ diâm., contendo micélio, foram armazenados em água destilada esterilizada e estocados a $4{ }^{\circ} \mathrm{C}$.

Caracterização morfológica - As características de crescimento da colônia em BDA foram avaliadas quanto às colorações (branca, creme ou castanho), o aspecto da colônia (grumoso, aveludado ou liso), a abundância de micélio aéreo (abundante ou escasso). Foram feitas medições dos diâmetros das hifas vegetativas (Currah 1986; Currah et al. 1987; 1989; 1997; Currah \& Zelmer 1992; Pereira et al. 2003a; 2005; Zelmer \& Currah 1995).

Para estimar-se a taxa diária de crescimento das colônias em meio fubá ágar (FA) (Pereira et al. 2003a) e BDA, discos de micélio de $9 \mathrm{~mm}$ diâm. retirados das bordas das colônias dos isolados crescidos nos respectivos meios de cultura, foram transferidos para o centro da placa contendo $20 \mathrm{~mL}$ destes meios e incubados a $28{ }^{\circ} \mathrm{C}$. Para cada espécie foi medido diariamente o diâmetro da colônia nos dois diferentes meios, sendo que, para cada meio foram feitas quatro repetições.

Coloração de núcleos - Para a coloração de núcleos, fragmentos do micélio dos isolados, crescidos por 10 dias a $28^{\circ} \mathrm{C}$, foram montados entre lâmina e lamínula com $50 \mu \mathrm{L}$ de uma solução corante contendo $1 \mu \mathrm{L} / \mathrm{mL}$ SYBR ${ }^{\circledR}$ Green em $\mathrm{KH}_{2} \mathrm{PO}_{4} 10 \mathrm{mM}$ e glicerol a $18 \%$ (Meinhardt et al. 2001). Após cinco minutos, em câmara escura, as lâminas foram observadas em microscópio óptico Nikon E 600 acoplado de epifluorescência Y-FL operando na faixa de comprimento de onda de 450-520 nm de excitação.

Identificação dos isolados - Para a identificação dos isolados seguiu-se a proposição de Moore (1987) e de Currah \& Zelmer (1992), a qual se baseia nas características morfológicas, adotando-se a chave e notas para fungos micorrízicos em orquídeas.

\section{Resultados e discussão}

Os isolamentos preliminares apresentaram melhores resultados quando se utilizaram plantas jovens ou "seedlings". Em plantas adultas ou com raízes muito envolvidas por matéria orgânica, o número de isolados de fungos endofíticos não rizoctonióides foi alto. Dentre estes destacaram-se os pertencentes ao grupo Mycelium radicis atrovirens (Melin 1922), atualmente denominados de "Dark Septate Endophytes (DSE)" (Jumpponen \& Trape 1998). Por outro lado, o fato de muitas plantas que ocorrem em campos rupestres crescerem diretamente sobre a superfície rochosa (canga ou quartzito) pode ter favorecido o isolamento, pois suas raízes encontravam-se livres de matéria orgânica em decomposição.

O sucesso no isolamento foi obtido para as oito espécies de orquídeas, sendo que quatro ocorriam sobre canga e quatro sobre quartzito (Tab. 1). Os gêneros Rhizoctonia, Epulorhiza e Ceratorhiza foram descritos em associação a orquídeas em campos rupestres do Brasil, sendo que todos ocorreram tanto em canga quanto em quartzito (Tab. 1). O gênero Rhizoctonia está representado por dois isolados OM 19 e OM 21, sendo que o primeiro foi isolado de planta que crescia sobre canga, e o segundo, sobre quartzito. O gênero Epulorhiza está representado pelos isolados de orquídeas OM 18 e OM 20 que ocorrem sobre quartzito e canga, respectivamente. O gênero Ceratorhiza está representado pelos isolados OM 11, OM 13, OM 14 e OM 15, os dois primeiros associados a orquídeas sobre quartzito e os outros dois sobre canga.

A caracterização dos isolados (Tab. 2) demonstrou diferenças marcantes entre os isolados do gênero Epulorhiza em relação aos gêneros Rhizoctonia e Ceratorhiza, principalmente no que diz respeito ao aspecto da colônia (Fig. 1), taxa diária de crescimento (Tab. 2), morfologia das células monilióides (Fig. 2 a 6) e formação de pseudoescleródios (Fig. 7 e 8). A medição do diâmetro das hifas também mostrou-se de grande importância nesta diferenciação (Tab. 2). As diferenças visuais entre os isolados de Rhizoctonia e Ceratorhiza não foram muito marcantes. No entanto, com a análise da condição nuclear foi possível fazer a distinção entre os gêneros (Tab. 2).

A condição multinucleada dos isolados, aqui considerados como Rhizoctonia, poderiam ser classificados como pertencentes ao gênero Moniliopsis (Moore 1985). Moore (1987) propõe o gênero Moniliopsis para acomodar anamorfos com teleomorfos em Thanatephorus e Waitea, ambos com células multinucleadas. Entretanto, apesar da proposta estar de acordo com o Código Internacional de Nomeclatura Botânica, a denominação Rhizoctonia foi mantida (Nomina Conservanda) em razão da 


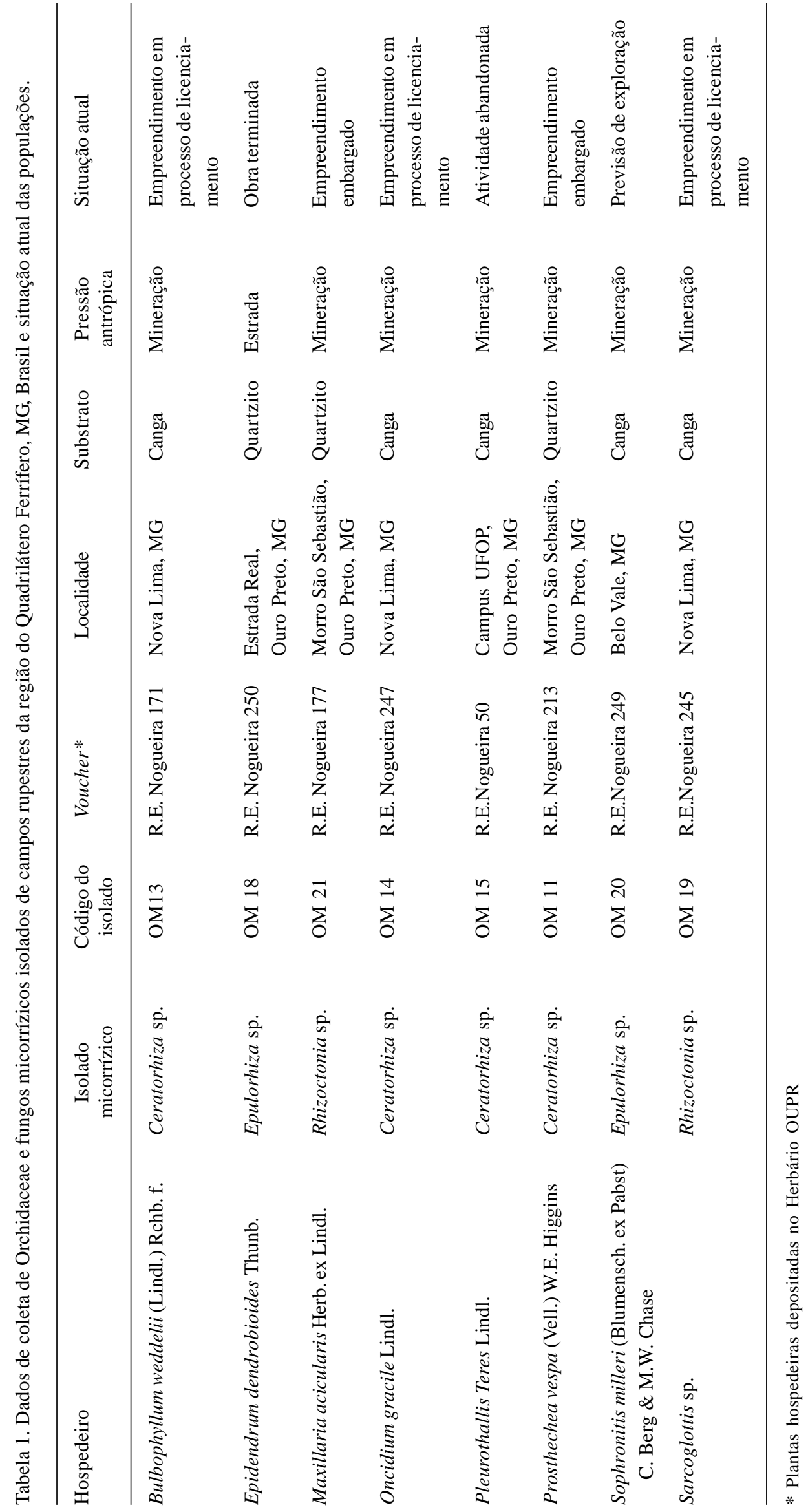



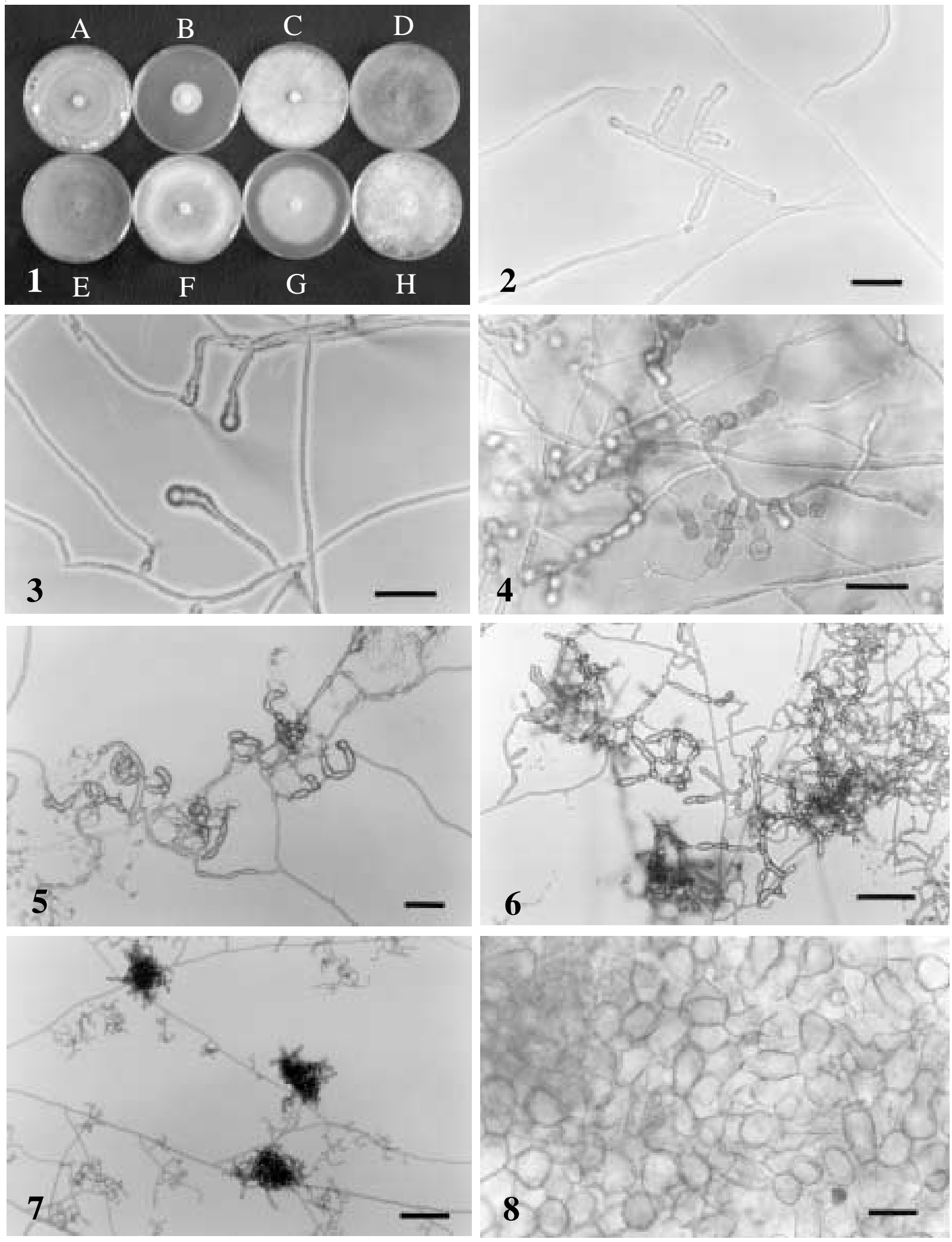

Figuras 1-7. 1. Culturas puras dos fungos: (A) OM13; (B) OM18; (C) OM2; (D) OM14; (E) OM15; (F) OM11; (G) OM20 e (H) OM19. 2. OM13, hifas em fase de formação de células monilióides, verifica-se ângulo de $90^{\circ}$ entre as ramificações (barra $\left.=50 \mu \mathrm{m}\right)$. 3. OM20, início da formação de células monilióides (barra $=40 \mu \mathrm{m})$. 4. OM20, cadeia de células monilióides $($ barra $=40 \mu \mathrm{m})$. 5. OM11, células monilióides (barra $=50 \mu \mathrm{m})$. 6. OM14, células monilióides (barra $=50 \mu \mathrm{m})$. 7. OM15, início da formação de pseudoescleródio (barra $=100 \mu \mathrm{m}) .8$. OM15, corte de pseudoescleródio bem desenvolvido (barra $=25 \mu \mathrm{m})$. 
Tabela 2. Características morfológicas e de coloração usadas para a diferenciação e identificação dos isolados de fungos micorrízicos em Orchidaceae de campos rupestres na região do Quadrilátero Ferrífero, MG, Brasil. BDA = batata-dextrose-ágar; FA = fubá-ágar.

\begin{tabular}{|c|c|c|c|c|c|c|c|}
\hline \multirow[t]{2}{*}{ Cód.** } & \multicolumn{2}{|c|}{ Taxa de crescimento } & \multirow[t]{2}{*}{ Cor } & \multirow[t]{2}{*}{ Micélio aéreo } & \multirow[t]{2}{*}{ Aspecto } & \multirow[t]{2}{*}{ Condição nuclear } & \multirow[t]{2}{*}{ Diâmetro das hifas } \\
\hline & BDA & FA & & & & & \\
\hline OM13 & $0,60 *$ & 0,65 & branco & abundante & floculado & binucleado & $>4 \mu \mathrm{m}$ \\
\hline OM 18 & 0,06 & 0,12 & branco & escasso & mucoso & binucleado & $<4 \mu \mathrm{m}$ \\
\hline OM 21 & 0,95 & 1,07 & branco & abundante & liso & multinucleado & $>4 \mu \mathrm{m}$ \\
\hline OM 14 & 1,75 & 1,28 & marrom & abundante & grumoso & binucleado & $>4 \mu \mathrm{m}$ \\
\hline OM 15 & 1,45 & 1,32 & marrom & abundante & grumoso & binucleado & $>4 \mu \mathrm{m}$ \\
\hline OM 11 & 0,67 & 0,74 & branco & abundante & aveludado & binucleado & $>4 \mu \mathrm{m}$ \\
\hline OM 20 & 0,31 & 0,43 & branco & escasso & mucoso & binucleado & $<4 \mu \mathrm{m}$ \\
\hline OM 19 & 1,49 & 1,02 & branco & abundante & aveludado & multinucleado & $>4 \mu \mathrm{m}$ \\
\hline
\end{tabular}

* cm/dia;** Código de identificação do isolado conforme Tab. 1.

importância econômica desse táxon e do número de trabalhos já publicados utilizando-se esta terminologia (Stalpers et al. 1998).

Quanto à análise dos microhábitats dentro das áreas de ocorrência das populações de orquídeas, houve uma amostragem considerável, pois foram contempladas no estudo: plantas rupícolas ( $P$. vespa, $M$. acicularis, O. gracile, $P$. teres, B. weddelii e $S$. milleri), uma planta aquática (E. dendrobioides) e uma terrestre (Sarcoglottis sp.). Isto demonstra que a versatilidade da família Orchidaceae na exploração de nichos bastante distintos pode estar relacionada à presença dos fungos simbiontes nos diferentes hábitats, como já relatado para orquídeas de regiões temperadas ou subtropicais (Rasmussen \& Whigham 1998).

O fato das espécies de orquídeas estudadas não pertencerem ao mesmo gênero dificulta o entendimento das relações de especificidade fungoplanta. No entanto, demonstra-se mais uma ocorrência de Epulorhiza em plantas do gênero Epidendrum, como já relatado para E. conopseum e E. rigidum (Zettler et al. 1998; Pereira et al. 2003a). Além disso, a espécie $E$. dendrobioides possui hábitat aquático, diferente daqueles das espécies até então estudadas, sugerindo que a ocorrência de Epulorhiza em Epidendrum pode estar influenciada mais pela especificidade fungo-hospedeiro do que pelo hábitat da planta.

Áreas degradadas por atividades de mineração têm a composição microbiológica do solo alterada, como já demonstrado em estudos com outros tipos de fungos micorrízicos (Silva et al. 2001). A ocorrência de Pleurothallis teres em uma área onde já funcionou uma mina de bauxita e que ainda sofre pressões antrópicas, além de estar de acordo com a hipótese de que esta espécie participa do processo de sucessão primária em áreas de afloramentos rochosos (Alves et al. 2000), sugere a possibilidade de utilização do fungo micorrízico isolado de seu sistema radicular para o manejo de orquídeas ameaçadas ou extintas localmente.

Dentre os isolados, o OM 20 (Epulorhiza sp.) tem grande importância, pois sua planta hospedeira (Sophronitis milleri) teve seu hábitat totalmente destruído por mineradoras e suas populações dizimadas por mateiros e orquidófilos, isto devido ao grande atrativo de suas flores vermelhas. Estratégias que visem a recuperação das populações de orquídeas ameaçadas devem utilizar o uso da germinação simbiótica (Zettler \& Hofer 1998; Zettler et al. 2001), pois os ciclos de vida das espécies de orquídeas estão determinados, na natureza, pela presença de um fungo micorrízico simbionte.

Os isolados do gênero Rhizoctonia (OM 19 e OM 21) ocorrem associados a uma espécie terrestre do gênero Sarcoglottis e à espécie Maxillaria acicularis (Tab. 1). Estas têm, em seus microhábitats, substrato rico em matéria orgânica. Sarcoglottis sp. habita fendas preenchidas por material proveniente da degradação da canga e da biodegradação da flora rupestre. Maxillaria acicularis, apesar de crescer sobre grandes placas de quartzito, possui propagação vegetativa considerável e retêm a matéria orgânica em seus rizomas. Logo, assim como em outros trabalhos (Masuhara \& Katsuya 1994) os fungos micorrízicos do gênero Rhizoctonia são comumente encontrados em microhábitats terrestres ou ricos em matéria orgânica.

Os isolados do gênero Ceratorhiza (OM 11, OM 13 e OM 14) estão associados a espécies que crescem 
diretamente sobre a rocha: Prosthechea vespa, Bulbophyllum weddelii e Oncidium gracile (Tab. 1). Sabe-se que nestes ambientes há variação muito grande nas condições de temperatura e umidade (Giulietti \& Pirani 1988). Ao contrário dos isolados OM 19 e OM 21, os isolados OM 11, OM 13 e OM 14 estão associados a microhábitats com baixíssima quantidade de matéria orgânica em decomposição. Estas observações indicam que pode ocorrer uma diferença na composição taxonômica de fungos micorrízicos orquidóides, em diferentes microhábitats, dentro de áreas de campos rupestres.

Em conclusão, orquídeas em campos rupestres ocorrem associadas a fungos rizoctonióides micorrízicos dos gêneros Epulorhiza, Ceratorhiza e Rhizoctonia. Entretanto, ainda não foi possível determinar padrões que expliquem a distribuição destes fungos ou as suas relações de especificidade com os hospedeiros. $\mathrm{O}$ fato de alguns isolados ocorrerem em áreas muito impactadas, aponta para a possibilidade de uso dos mesmos na recomposição destas e de outras áreas degradadas por mineradoras no Quadrilátero Ferrífero, estratégia já utilizada em regiões temperadas para a reintrodução de espécies ameaçadas (Zettler \& Mcinnis 1992).

As técnicas tradicionalmente usadas para a propagação comercial de orquídeas não preservam os microrganismos e, muitas vezes, fazem o uso de plantas clonadas. Estudos sobre germinação simbiótica usando espécies nativas com potencial de exploração comercial são necessários, pois esta técnica possibilitará maior conservação da diversidade desses fungos micorrízicos (Pereira et al. 2003b).

Pereira et al. (2003a) relataram o primeiro fungo micorrízico rizoctonióide isolado de orquídeas epífitas no Brasil. Este trabalho vem contribuir no conhecimento de fungos micorrízicos de orquídeas no Brasil, além de contribuir como parte da micoteca do Laboratório de Associações Micorrízicas DMB/ UFVB. Novos trabalhos devem ser direcionados à germinação simbiótica, ao estudo da especificidade fungo-hospedeiro e à distribuição dos fungos nos hábitats. Espera-se que a continuidade destes trabalhos possibilite o desenvolvimento de tecnologia de manejo e exploração sustentável da nossa flora.

\section{Referências bibliográficas}

Alves, R.J. \& Kolbek, J. 2000. Primary succession on quartzite cliffs in Minas Gerais, Brasil. Biologia Bratislava 55(1): 69-83.
Arditti, J.; Ernst, R.; Yam, T.W. \& Glabe, C. 1990. The contributions of orchid mycorhrizae fungi to seed germination: a speculative review. Lindleyana 5(4): 249-255.

Clements, M.A. 1988, Orchid micorrhizal associations. Lindleyana 3(2): 73-86.

Costa, C.M.R.; Herrmann, G.; Martins, C.S.; Lins, L.V. \& Lamas, I.R. 1998. Biodiversidade em Minas Gerais - Um atlas para sua conservação. Belo Horizonte, Fundação Biodiversitas.

Currah, R.S. 1986. Thanatephorus pennatus sp. nov. isolated from mycorrhizal roots of Calypso bulbosa (Orchidaceae) from Alberta. Canadian Journal of Botany 65: 1957-1960.

Currah, R.S.; Sigler, L. \& Hambleton, S. 1987. New records and new taxa of fungi from the micorrhizae of terrestrial orchids of Alberta. Canadian Journal of Botany 65: 2473-2481.

Currah, R.S.; Smereciu, E.A. \& Hambleton, S. 1989. Mycorrhizae and mycorrhizal fungi of boreal species of Plantanthera and Coeloglossum (Orchidaceae). Canadian Journal of Botany 68: 1171-1181.

Currah, R.S. \& Zelmer, C.D. 1992. A key and notes for the genera of fungi mycorrhizal with orchids and a new species in the genus Epulorhiza. Reports of the Tottori Mycological Institute 30: 43-59.

Currah, R.S.; Zettler, L.W. \& McInnis, T.M. 1997. Epulorhiza inquilina sp. nov. from Platanthera (Orchidaceae) and a Key to Epulorhiza species. Mycotaxon 61: 338-342.

Giulietti, A.M. \& Pirani, J.R. 1988. Patterns of geographic distribution of some plant species from the Espinhaço Range, Minas Gerais and Bahia, Brazil. Pp. 39-69. In: W. R. Heyer \& P. E. Vanzolini (eds.). Proceedings of a workshop on Neotropical distribution patterns. Rio de Janeiro, Academia Brasileira de Ciências.

Jumpponen, A. \& Trappe, J.M. 1998. Dark Septate Endophytes: a review of facultative biotrophic rootcolonizing fungi. New Phytologist 140: 295-310.

Marx, D.H. 1969. Antagonism of mycorrhizal fungi to root pathogenic and soil bacteria. Phytopatology 59: 153-163.

Masuhara, G. \& Katsuya, K. 1994. In situ and in vitro specificity between Rhizoctonia spp. and Spiranthes sinensis (Persoon) Ames. var. amoena (M. Bieberstein) Hara (Orchidaceae). New Phytologist 127: 711-718.

Meinhardt, L.W.; Bellato, C.M. \& Tsai, S.M. 2001. SYBRâ Green I used to evaluate nuclei number of fungal mycelia. Biotechniques 31: 42-46.

Melin, E. 1922. On the mycorrhizas of Pinus silvestris L. and Picea abies Karst. Journal of Ecology 9: 254-257.

Menezes, N.L. \& Giulietti, A.M. 1986. Serra do Cipó, Paraíso dos botânicos. Ciência Hoje 4: 38-44.

Moore, R.T. 1985. The challenge of the dolipore/ parenthesome septum. Pp. 175-212. In: D. Moore; L.A. Casselton; D.A. Wood \& J. Frankland (eds.). Developmental Biology of Higher Fungi. Cambridge, Cambridge University Press.

Moore, R.T. 1987. The genera of Rhizoctonia-like fungi: Ascorhizoctonia, Ceratorhiza gen. nov., Epulorhiza gen. nov., Moniliopsis and Rhizoctonia. Mycotaxon 29: 91-99. 
Pereira, O.L.; Kasuya, M.C.M.; Borges, A.C. \& Araújo, E.F. 2005. Morphological and molecular characterization of mycorrhizal fungi isolated from neotropical orchids in Brazil. Canadian Journal of Botany 83: 54-65.

Pereira, O.L.; Rollemberg, C.L.; Borges, A.C.; Matsuoka, K. \& Kasuya, M.C.M. 2003a. Epulorhiza epiphytica sp. nov. isolated from mycorrhizal roots of epiphytic orchids in Brazil. Mycoscience 44:153-155.

Pereira, O.L.; Rollemberg, C.L. \& Kasuya, M.C.M. 2003 b. Association des mycorhizies dans les orchidees perspectives d'utilisation dans les programmes de propagation symbiotique. Orchidees 55: 24-27.

Peterson, R.L.; Uetake, Y. \& Zelmer, C. 1998. Fungal symbioses with orchid protocorms. Symbiosis 25: 29-55.

Rasmussen, H.N. 1995. Terrestrial Orchids: From Seed to Mycotrophic Plant. Cambridge, Cambridge University Press.

Rasmussen, H.N. \& Whigham, D.F. 1998. Importance of woody debris in seed germination of Tipularia discolor (Orchidaceae). American Journal of Botany 85: 829-834.

Richardson, K.A. \& Currah, R.S. 1995. The fungal community associated with the roots of some rainforest epiphytes of Costa Rica. Selbyana 16: 49-73.

Richardson, K.A.; Currah, R.S. \& Hambleton, S. 1993. Basidiomycetes endophytes from the roots of neotropical epiphytic orchidaceae. Lindleyana 8: 127-137.

Silva, G.A. 2001. Potencial de infectividade de fungos micorrízicos arbusculares oriundos de área de caatinga nativa e degradada por mineração no Estado da Bahia, Brasil. Revista Brasileira de Botânica 24: 135-143.
Smith, S.E. 1966. Physiology and ecology of orchid mycorrhizal fungi with reference to seedling nutrition. New Phytologist 65: 488-499.

Stalpers, J.A.; Andersen, T.F. \& Gams, W. 1998. Two proposals to conserve the names Rhizoctonia and $R$. solani (Hyphomycetes). Taxon 47: 725-726.

Zelmer, C.D. \& Currah, R.S. 1995. Ceratorhiza pernacatena and Epulorhiza calendulina spp. nov.: mycorrhizal fungi of terrestrial orchids. Canadian Journal of Botany 73: 1981-1985.

Zettler, L.W.; Delaney, T.W. \& Sunley, J.A. 1998. Seed propagation of the epyphytic green-fly orchid, Epidendrum conopseum R. Brown, using its endophytic fungus. Selbyana 19: 249-253.

Zettler, L.W. \& Hofer, C.J. 1998. Propagation of the little club-spur orchid (Platanthera clavelata) by symbiotic seed germination and its ecological implications. Environmental and Experimental Botany 39: 189-195.

Zettler, L.W. \& Mcinnis, T.M. 1992. Propagation of Platanthera integrilabia (Correll) Luer, an endangered terrestrial orchid, through symbiotic seed germination. Lindleyana 7: 154-161.

Zettler, L.W.; Stewart, S.L.; Bowles, M.L. \& Jacobs, K.A. 2001. Mycorrhizae fungi and cold-assisted symbiotic germination of the federally threatened eastern prairie fringed orchid, Platanthera leucophala (Nuttall) Lindley. American Midland Naturalist 145: 168-175. 